\title{
28 Research Suare \\ Gut microbial diversity and composition of Indian white shrimp (Penaeus indicus)
}

\section{Prasanna Kumar Patil}

ICAR-Central Institute of Brackishwater Aquaculture

\section{Vinay TN ( $\sim$ vinaytn56@gmail.com )}

ICAR - Central Institute of Brackishwater Aquaculture https://orcid.org/0000-0001-6863-1552

\section{Sudeep Darbhe Ghate}

Central Research Laboratory, KSHEMA, NITTE

\section{Viswanathan Baskaran}

ICAR-Central Institute of Brackishwater Aquaculture

\section{Satheesha Avunje}

ICAR-Central Institute of Brackishwater Aquaculture

\section{Research Article}

Keywords: Aquaculture, Indian white shrimp (Penaeus indicus), Gut microbiota, MOTHUR

Posted Date: August 13th, 2021

DOI: https://doi.org/10.21203/rs.3.rs-807775/v1

License: (c) (1) This work is licensed under a Creative Commons Attribution 4.0 International License. Read Full License 


\section{Abstract}

The endemic Indian white shrimp (Penaeus indicus) is an economically important shrimp species, distributed in the Indo-West Pacific region. Knowledge of gut microbial composition helps in dietary interventions to ensure improved health and production. Here we analyzed V3-V4 hypervariable regions of the $16 \mathrm{~S}$ rRNA gene to examine intestinal microbiota in the wild and farmed $P$. indicus. The study revealed Proteobacteria, Fusobacteria, Tenericutes, and Bacteroidetes, were the dominant phyla in both the groups; though the differences in relative abundance were evident. The dominant genera in case of the wild group were Photobacterium (29.5\%) followed by Propionigenium (13.9\%), Hypnocyclicus (13.7\%) and Vibrio (11.1\%); while Vibrio (46.5\%), Catenococcus (14\%), Propionigenium (10.3\%) and Photobacterium (8.7\%) were dominant in the farmed group. The results of the study suggest the role of environment on the relative abundance of gut bacteria. This is the first report characterizing gut microbial diversity in $P$. indicus, which can be used to understand the role of gut microbiota in health, nutrition, reproduction, and growth.

\section{Introduction}

Gut microbiota is known to play an important role in host development, health and growth of various terrestrial and aquatic animals (Nelson et al. 2013; Kho and Lal 2018; Li et al. 2018; Butt and Volkoff 2019). Continuous efforts are being made to enhance the knowledge of microbiota-host interactions in aquatic animals. The gut microbiota of commercially important shrimps, Penaeus merguiensis (Oxley et al. 2002), P. chinensis (Liu et al. 2011) and P. penicillatus (Wang et al. 2014), have been reported from wild and farmed environments using traditional culture-dependent approaches. Recently, high throughput sequencing approach was applied to understand the gut microbiota of $P$. vannamei (Chen et al. 2017; Cornejo et al. 2017; Zeng et al. 2017; Li et al. 2018; Md-Zoqratt et al. 2018; Fan and Li 2019; Fan et al. 2019; Gao et al. 2019), P. monodon (Chaiyapechara et al. 2012; Rungrassamee et al. 2013, 2014, 2016) and $P$. stylirostris (Cardona et al. 2016) from farmed, wild, diseased and grown under different environmental conditions.

The Indian white shrimp ( $P$. indicus) is endemic to Indo-West Pacific region, and it is an economically important shrimp species for aquaculture in India and the Middle East (CIBA Annual report 2017, Sajeela et al. 2019). P. indicus was the primary shrimp species for aquaculture in the region before adopting $P$. monodon (Black tiger shrimp) for its higher productivity (CIBA Annual report 2017) followed by specific pathogen-free (SPF) and selectively bred P. vannamei (Pacific white shrimp) since 2010 (Remany et al. 2010). At present, shrimp farming is dominated by a single species, $P$. vannamei. Species diversification is essential for sustainable farming activity (Metian et al. 2020); hence, attempts are underway in India to revive the culture of $P$. indicus through improved emphasis on captive breeding, seed production and intensive farming. The large-scale demonstration of $P$. indicus farming has been successfully conducted using hatchery-produced seeds in the country (CIBA Annual report 2017). 
Specific gut microbiota has been reported to influence the reproductive fitness of the captive broodstock of black rhino (Antwis et al. 2019), indicating the role of microbiota in reproductive activities.

Identification of such biomarkers helps in developing breeding plans and production of healthy offspring in penaeid shrimps. The early stages of larval development face challenges with bacterial infections leading to mortalities in hatcheries and grow-out farms (Vandenberghe et al. 1999; Zhou et al. 2012). Following the regulatory restrictions on the use of undesired pharmacologically active substances in aquaculture, the use of beneficial microbes to manipulate the intestinal microbiota was suggested (Nayak 2010). Hence, maintaining the right balance of gut microbiota is crucial for the overall health of the host. Presently, there are no reports on the gut microbiota of $P$. indicus. Analysis of the gut microbiome of $P$. indicus (wild and farmed) may provide crucial information on the microbial diversity and composition.

With the emergence of next-generation sequencing (NGS) technologies, 16S rRNA high throughput sequencing has become more comprehensive culture-independent approach to study gut microbiota (Tarnecki et al. 2017). In this study, we have used 16S rRNA high throughput sequencing to study the composition of gut bacterial communities of $P$. indicus. This study will be the preliminary understanding for further deeper investigations.

\section{Materials And Methods}

\subsection{Shrimp and intestinal sample collection}

P. indicus ( $\mathrm{n}=15,12.83 \pm 1.37 \mathrm{~g}$ ) were collected from the ponds of domestication and captive breeding program facility at the Muttukadu Experimental Station of ICAR-Central Institute of Brackishwater Aquaculture, reared at a stocking density of $30 / \mathrm{m}^{2}$, fed with an artificial diet containing $35 \%$ protein. Wild $P$. indicus $(\mathrm{n}=15,13.11 \pm 1.82 \mathrm{~g})$ were collected from the Bay of Bengal near Kalpakkam, Chennai, Tamil Nadu (12030'48.5" N 80009'54.6" E) region in June 2019. Both the groups were shifted to the laboratory and maintained in seawater ( $35 \mathrm{ppt}$ ) for $24 \mathrm{~h}$ without feeding. Shrimps with empty gut were washed thoroughly in sterile seawater, and the surface was disinfected with $70 \%$ alcohol and the whole intestine was aseptically removed and used for DNA extraction.

\subsection{DNA Extraction, library preparation and sequencing}

Intestine from five shrimp was pooled to avoid individual bias. Genomic DNA from the intestine samples was extracted using the QIAamp DNA stool mini kit (Qiagen, Germany) according to the manufacturer's protocol. The DNA concentration and purity were determined using NanoDrop ND-1000 spectrophotometer (Thermo Scientific, USA). Three samples each from wild and farmed shrimp were subjected for PCR amplification and next-generation sequencing (Eurofins Genomics and Bioinformatics Laboratory, India). The 16S rRNA V3-V4 hypervariable region was targeted using primers $16 \mathrm{~S} F$ GCCTACGGGNGGCWGCAG and 16S R ACTACHVGGGTATCTAATCC to profile bacterial communities. The amplicon libraries were prepared using the Nextera XT index kit (Illumina Inc. USA) as per metagenomic sequencing library preparation protocol and sequenced using Illumina MiSeq platform. 


\subsection{Data analysis}

The raw sequences were processed using MOTHUR pipeline (v. 1.42) (Schloss et al. 2009) to filter reads, create contigs; reduce noise as per standard MiSeq procedure. Sequences were aligned, clustered and identified taxonomically with SILVA database (http://arb-silva.de) release 132. Chimera.vsearch option was used to identify and remove chimeras. Sequences with $97 \%$ identity threshold were classified into operational taxonomic units (OTUs) at genetic distances of 0.03 . Taxonomy was assigned by comparing to the reference database (Schloss et al. 2009; Wang et al. 2007) and aligned using the align.seqs command in MOTHUR. Sequences were rarefied before calculation of alpha and beta diversity statistics. Alpha diversity indexes were calculated from rarefied samples using calculators for richness and diversity indices of the bacterial community (i.e., sobs, Chao, Shannon, Simpson, and ACE). Venn diagram was prepared using a web-based tool, interactiVenn (Heberle et al. 2015). Non-parametric t-test was carried out using linear discriminant analysis (LEfSe) to determine the significantly differing OTU's between the groups. The data output was subjected to detailed statistical and meta-analysis using an online tool MicrobiomeAnalyst (Dhariwal et al. 2017) for visualization.

\section{Results}

\subsection{Characteristics of $16 \mathrm{~s}$ rRNA sequencing}

Microbiota of $P$. indicus was analyzed by sequencing 16S rRNA gene (V3-V4 hypervariable regions) using Illumina MiSeq platform and was processed using MOTHUR pipeline. After filtering and removal of chimera and undesirable sequences, a total of 598214 high-quality reads were obtained from six samples with an average of 99702 reads, ranging from 67571 to 114392 . A total of 4502 OTU's were obtained at a sequence similarity of $97 \%$. The rarefaction curves for the OTU's detected in this study showed that the quality of observed species increased with the depth of sequencing (Figure S1). Good's coverage estimator for all samples was $>99.1 \%$, indicating the maximum coverage and the samples were represented sufficiently. Detected bacteria in both the samples (wild and farmed) were classified into 31 phyla, 72 classes, 187 orders, 324 family and 588 genera. $0.08 \%$ of the bacteria in farmed and $0.14 \%$ in wild samples were unclassified.

\subsection{Diversity analysis of microbiota in wild and aquacultured $P$. indicus}

Diversity indices provide crucial quantitative information about rarity and commonness of species which helps to understand community structure. We measured a-diversity (Chao 1, ACE, Shannon and Simpson) indices of wild-caught and farmed $P$. indicus microbiota. The a-diversity indices showed significant differences between the wild and farmed group. The wild samples showed increased richness (Chao and ACE) and evenness (Shannon, Simpson) compared to farmed group (Fig. 1). There were no significant differences in the homogeneity (within-group similarity) and composition of microbiota between wild and farmed shrimp. 


\subsection{Comparison of gut microbiota between wild and aquacultured $P$. indicus}

The most abundant phylum commonly present in $P$. indicus from wild and aquaculture origin were represented mainly by Proteobacteria and Fusobacteria while Tenericutes, Firmicutes, Bacteroidetes, Planctomycetes and Cyanobacteria were in lesser abundance (Fig. 2a). At generic level, Vibrio, Photobacterium, Catenococcus, Propionigenium and Hypnocyclicus were highly abundant (Fig. 2b) which formed the core microbiota. The shared taxa in both the groups may be considered as core gut microbiota and 329 OTUs were shared between wild and aquacultured shrimp (Fig. 3), while 71 were unique to wild and 30 were unique to farmed group.

\subsection{Composition of microbial communities in wild and aquacultured $P$. indicus}

The relative abundance data of farmed $P$. indicus showed differences from that of wild and Fig. 4a show the relative abundance of top ten phyla. In wild shrimp the five most abundant phyla (>90\%) were Proteobacteria (58.64\%), Fusobacteria (27.75\%), Bacteroidetes (4.57\%), Planctomycetes $(2.23 \%)$ and Epsilonbacteraeota (1.90\%); whereas in farmed shrimp, the five most abundant phyla $(>90 \%)$ were Proteobacteria (77.27\%), Fusobacteria (12.88\%), Tenericutes (5.38\%), Firmicutes (1.79\%) and Cyanobacteria (1.16\%).

At genus level, the wild $P$. indicus microbiota mainly consisted of Photobacterium (29.5\%), Propionigenium (13.9\%), Hypnocyclicus (13.7\%), Vibrio (11.1\%), Catenococcus (9.9\%) and Tenacibaculum (3.3\%); whereas Vibrio (46.5\%), Catenococcus (14.0\%), Propionigenium (10.3\%), Photobacterium (8.7\%), Candidatus_Hepatoplasma (5.3\%), Gammaproteobacteria_unclassified (4.3\%) and Hypnocyclicus (2.5\%) were the most abundant genus in aquacultured shrimp (Fig. 4b).

Linear discriminant analysis effect size (LEfSe) was performed to identify the specific taxa significantly varied in abundance in with wild or farmed shrimp. The significant differences were observed in the phylum Proteobacteria, Tenericutes, Firmicutes, Kiritimatiellaeota, Planctomycetes, Dadabacteria, Chlamydiae. In total, 30 genera, varying significantly were identified with LDA scores > 3 (Figure S2). Genus Vibrio was significantly associated with the farmed group, while Photobacterium was significantly associated with the wild group.

\section{Discussion}

Information on gut microbial diversity of a commercially farmed seafood species will help in devising suitable intervention strategies for enhanced health, growth and production. The gut microbiota is an evolving system ( $\mathrm{Li}$ et al. 2017) and the abundance and species diversity will play a significant role in host development, physiology, immune system and growth (Ramírez et al. 2017, 2017a). Considering the lack of data, wild and farmed $P$. indicus were analyzed for gut microbiota. This is the first characterization 
of gut microbiota in Indian white shrimp ( $P$. indicus) employing 16S rRNA high-throughput sequencing. The culture-independent methods are more reliable to characterize the bacterial diversity than the culture dependent methods, as majority (98-99\%) of the bacteria are unculturable and those obtained by culture-based methods are not a true representative of the bacterial diversity (Ringo et al. 2016; Qiao et al. 2017; Suo et al. 2017).

The results obtained in this study were consistent with other aquatic organisms in having a sizeable bacterial diversity (Ramírez et al. 2017; Wu et al. 2012). The Good's coverage was $>99.1 \%$, suggesting the maximum coverage and complete identification of bacterial communities in all the samples.

The core gut microbiota, which forms $>90 \%$ of the bacterial diversity in $P$. indicus from wild were mainly classified into Proteobacteria, Fusobacteria, Bacteroidetes, Planctomycetes and Epsilonbacteraeota at the phylum level. At the same time, farmed shrimp have a core microbiota comprising of Proteobacteria, Fusobacteria, Tenericutes, Firmicutes and Cyanobacteria. Abundance of Proteobacteria observed in the present study was consistent with the reported results from other important penaeid shrimps $P$. vannamei (Chen et al. 2017; Cornejo et al. 2017; Zeng et al. 2017; Li et al. 2018; Md-Zoqratt et al. 2018; Fan and Li 2019; Fan et al. 2019; Gao et al. 2019), P. monodon (Chaiyapechara et al. 2012; Rungrassamee et al. $2013,2014,2016)$ and $P$. stylirostris (Cardona et al. 2016) and other aquatic organisms (Roeselers et al. 2011; Wu et al. 2012). Several studies in $P$. vannamei shows that Proteobacteria remains dominant, despite dietary modifications and physiological stress (Li et al. 2018). Though prevalence was similar, variation in the abundance of different phyla was observed between the shrimp groups. The microbiota will change according to the changes accompanying host development, diet, stress, and disease status and host phylogeny (Cardona et al. 2016; Yan et al. 2016). The artificial environment in farming system having different parameters from that of the natural environment might have led to the variation in the abundance of gut microbiota. The wild $P$. indicus mainly feed on gastropods, bivalves, crustaceans, polychaete, plant tissues, detritus etc. While, the aquacultured shrimp were fed with a formulated feed containing $35 \%$ proteins. Hence, the observed differences in the abundance of gut microbiota in the present study might be attributed to the change in environment, density of the animals and the diet.

Although Proteobacteria were abundant in both the group of shrimps, they were differentially distributed at the genus level. For example, the genus Vibrio is dominant (46.5\%) in farmed shrimp, while the wild harbored $11.1 \%$. In a similar study, Rungrassamee et al. (2014) reported a progressive increase in the abundance of Vibrio spp until 90 days (32.9\%) of observation in farm-reared $P$. monodon suggesting the role of environment and diet on the load of vibrios in the gut. On the contrary, a higher vibrio load in the wild $(20 \%)$ compared to the farmed (15.1\%) was reported in P. vannamei (Cardona et al. 2016). Rungrassamee et al. (2016) reported that, $P$. vannamei was able to restore normal gut microbiota and resist $V$. harveyi challenge compared to $P$. monodon. Vibrios are opportunistic pathogens of shrimps, which can cause up to $100 \%$ mortality (Liu et al. 2016). However, Zheng et al. (2017) demonstrated that all the dominating vibrio in the diseased shrimp were not pathogenic. Recently, Garibay-Valdez et al. (2018) reported that Vibrio spp maintain symbiotic relationship with host and gut microbiota in addition to modulating host signaling proteins, activate defense system and improve immune response like 
prophenoloxidase and superoxide dismutase activities (Ma et al. 2018; Qi et al. 2017; Velázquez et al. 2019). Further, Vibrios like $V$. gazogenes have been reported to confer resistance to bacterial infection suggesting their potential probiotic activity (Thompson et al. 2010). The higher abundance of Vibrio in healthy farmed shrimp may involve in these critical activities.

Followed by Vibrio, the genus Photobacterium was dominant among Proteobacteria and was abundant in gut of wild (29.5\%) compared to cultured (8.7\%) shrimp. Photobacterium is characterized as free-living marine commensals present in the gut of several marine organisms and reported as the indigenous bacterial population (Rungrassamee et al. 2014). Twenty species have been reported in the genus (Urbanczyk et al. 2011) and Photobacterium damselae sub sp. damselae is reported to be pathogenic to several marine finfish (Liu et al. 2016). Recently, Wang et al. (2019) suggested that Photobacterium play various roles in the health of $P$. vannamei. However, few reported the role of $P$. damselae subsp. Damselae in the black gill of $P$. monodon (Vaseeharan et al. 2007) and a symptom similar to AHPND in Exopalaemon carinicauda (Liu et al. 2016. The presence of Vibrio and Photobacterium in both wild and aquacultured shrimp gut indicates that they might be the common residents of $P$. indicus microbiota. Further efforts are required to study the association of these bacteria at different growth stages, and the effect of their dynamics on the health and metabolic functions.

We observed an abundance of Fusobacteria both in wild and farmed shrimp gut, and it was the second most dominant phyla; while their abundance was reportedly low in $P$. vannamei (Zhang et al. 2016) and $P$. monodon (Rungrassamee et al. 2014). Two genera, namely, Propionigenium and Hypnocyclicus, were dominant in this phylum, though their role in shrimp gut largely remains unknown. However, in a recent study, Propionigenium was suggested to play an essential role in limiting the WSSV infection in $P$. vannamei (Wang et al. 2019). Since our study reported an abundance of Fusobacteria, further investigation on their possible role in disease resistance will be of considerable significance to the aquaculture.

The phylum Bacteroidetes are known to improve the metabolism of polysaccharides, proteins, and other substances and enhance nutrient-utilization (Bäckhed et al. 2004) and promote the development of immune system (Hooper 2004). Significantly, lower abundance of these microbes in cultured animals $(0.41 \%)$ compared to the wild $(4.57 \%)$ suggest the possible opportunity for their inclusion in the dietary interventions for improved health and production in commercial shrimp operations. Though the pathogenic nature of these microbes in shrimp is not known, few reported the possible role in skin necrosis of marine fishes (Småge et al. 2016; Pérez et al. 2017; Frisch et al. 2018). Similar to previous observations (Cornejo et al. 2017; Zeng et al. 2017) the phylum Tenericutes were abundant (5.38\%) in cultured shrimp compared to that of wild $(0.18 \%)$. The abundance of genus Candidatus_Hepatoplasma in Tenericutes is correlated with the non-availability of food in ponds and suggested to play a role in host adaption to hunger situations (Fraune and Zimmer 2008; Wei et al. 2019). Additionally, several other phyla in low abundance may have a different role in the normal physiological function of the shrimp. 
Understanding the gut microbial profile has implications in aquaculture, and it is evident that gut microbiota largely depends on host phylogeny and diet. Maintaining near-natural gut microbiota has several advantages, and it is crucial for dietary alterations in commercial aquaculture practices. Adoption of beneficial microbes based on these studies could aid in sustainable development and improved productivity. The presently practiced dietary interventions through the application of probiotics, prebiotics, immunostimulants, antimicrobials etc. for enhanced health and production need to be evaluated, given their impact on gut microbial diversity for their practical utility.

\section{Conclusion}

This is the first report on the gut microbiota of Indian white shrimp, $P$. indicus, employing cultureindependent high throughput sequencing. The gut microbial profiles of wild-caught and farmed shrimp showed similarity in prevalence and differences in abundance. The results indicate the presence of possible host-associated resident bacterial populations while environment playing an essential role in differences in their abundance. However, $P$. indicus is distributed all along the coasts of Indian subcontinent and the inclusion of samples from all locations will enhance the resolution of gut microbiota of the species. Further studies on the gut microbiota at different growth stages and health status will help in devising intervention strategies for effective gut microbial modulations.

\section{Declarations}

\section{Acknowledgement}

The authors would like to extend a heartfelt thanks to all the staff of Crustacean culture division of ICARCIBA. The authors kindly acknowledge Dr. KK Vijayan, Director, CIBA, Dr. SV Alavandi, Principal Scientist and Head, AAHED, Dr. CP Balasubramanian, Principal Scientist and Head, CCD, CIBA for their support.

\section{Funding information}

This work was supported by Consortia Research Platform (CRP) on Vaccine and Diagnostics (CRP-V\&D) 1007611.

Authors contributions: Prasanna Kumar Patil and Vinay Tharabenahalli Nagaraju conceived and designed the project. Vinay Tharabenahalli Nagaraju collected the samples. Satheesha Avunje and Viswanathan Baskaran extracted the DNA and sequencing. Sudeep Darbhe Ghate and Vinay Tharabenahalli Nagaraju conducted bioinformatic analysis. Prasanna Kumar Patil, Vinay Tharabenahalli Nagaraju and Sudeep Darbhe Ghate analyzed the data, and wrote the paper. All authors reviewed and approved the manuscript.

Data availability: The raw sequences obtained in this study are available through the NCBI BioProject ID (PRJNA610456). 
Conflict of Interests: Authors declare no conflict of interest

Ethics Statement: The research undertaken complies with the current animal welfare laws in India. The experimental animal Penaeus indicus is not an endangered shrimp; the provisions of the Govt. of India's Wildlife Protection Act of 1972 are not applicable for experiments on this shrimp.

\section{References}

Antwis RE, Edwards KL, Unwin B, Walker SL, Shultz S (2019) Rare gut microbiota associated with breeding success, hormone metabolites and ovarian cycle phase in the critically endangered eastern black rhino. Microbiome 7: 27.

Bäckhed F, Ding H, Wang T, Hooper LV, Gou YK, Nagy A (2004) The gut microbiota as an environmental factor that regulates fat storage. PNAS USA 101: 15718-15723.

Butt RL, Volkoff H (2019) Gut microbiota and energy homeostasis in fish. Front. Endocrinol. 10: 9.

Cardona E, Gueguen Y, Magre K, Lorgeoux B, Piquemal D, Pierrat F, Noguier F, Saulnier D (2016) Bacterial community characterization of water and intestine of the shrimp Litopenaeus stylirostris in a biofloc system. BMC Microbiol. 16(1): 157.

Chaiyapechara S, Rungrassamee W, Suriyachay I, Kuncharin Y, Klanchui A, Karoonuthaisiri N, Jiravanichpaisal P (2012) Bacterial community associated with the intestinal tract of $P$. monodon in commercial farms. Microb. Ecol. 63: 938-953.

Chen WY, Ng TH, Wu JH, Chen JW, Wang HC (2017) Microbiome dynamics in a shrimp grow-out pond with possible outbreak of Acute Hepatopancreatic Necrosis Disease. Sci. Rep. 7: 9395.

CIBA Annual report 2016-2017 (2017) Central Institute of Brackishwater Aquaculture, Chennai, Tamil Nadu, India p. 36-40.

Cornejo-Granados F, Lopezzavala AA, Gallardobecerra L, Mendozavargas A, Sanchez F, Vichido R. et al. (2017) Microbiome of Pacific whiteleg shrimp reveals differential bacterial community composition between wild, aquacultured and AHPND/EMS outbreak conditions. Sci. Rep. 7: 11783.

Dhariwal A, Chong J, Habib S, King IL, Agellon LB, Xia J (2017) MicrobiomeAnalyst: a web-based tool for comprehensive statistical, visual and meta-analysis of microbiome data. Nuc. Acid Res. 45: 181-188.

Fan L, Li QX (2019) Characteristics of intestinal microbiota in the Pacific white shrimp Litopenaeus vannamei differing growth performances in the marine cultured environment. Aquaculture 505: 450-461.

Fan LF, Wang ZL, Chen MS, Qu YX, Li JY, Zhou AG, Xie SL, Zeng F, Zou JX (2019) Microbiota comparison of Pacific white shrimp intestine and sediment at freshwater and marine cultured environment. Sci. Tot. Environ. 657: 11941204. 
Fraune S, Zimmer M (2008) Host-specificity of environmentally transmitted mycoplasma-like isopod symbionts. Environ. Microbiol. 10(10): 2497-2504.

Frisch K, Småge SB, Johansen R, Duesund H, Brevik OJ, Nylund A (2018) Pathology of experimentally induced mouth rot caused by Tenacibaculum maritimum in Atlantic salmon smolts. PLoS One 13(11): e0206951.

Gao S, Pan L, Huang F, Song M, Tian C, Zang M (2019) Metagenomic insights into the structure and function of intestinal microbiota of farmed Pacific white shrimp (Litopenaeus vannamei). Aquaculture 499: 109-118.

Garibay-Valdez E, Martínez-Córdova LR, Vargas-Albores F, Gollas-Galván T, Lago-Leston A, Calderón K, Martínez-Porchas M (2018) Biofilm consumption shapes the intestinal microbiota of shrimp (Penaeus vannamei). Aquacult. Nutr. 25 (2): 427-435.

Heberle H, Meirelles GV, da-Silva FR, Telles GP, Minghim R (2015) InteractiVenn: a web-based tool for the analysis of sets through Venn diagrams. BMC Bioinfo. 16: 169.

Hooper LV (2004) Bacterial contributions to mammalian gut development. Trends Microbiol. 12: 129134.

Kho ZY, Lal SK (2018) The human gut microbiome-A potential controller of wellness and diseases. Front. Microbiol. 9: 1835.

Li E, Xu C, Wang X, Wang S, Zhao Q, Zhang Z, Qin GJ, Chen L (2018) Gut microbiota and its modulation for healthy farming of Pacific White Shrimp Litopenaeus vannamei. Rev. Fish. Sci. Aquacult. 26: 381-399.

Li Y, Hu X, Yang S, Zhou J, Zhang T, Qi L, Sun X, Fan M, Xu S, Cha M, Zhang M, Lin S, Liu S, Hu D (2017) Comparative analysis of the gut microbiota composition between captive and wild forest musk deer. Front. Microbiol. 8: 1705.

Liu F, Liu G, Li F (2016) Characterization of two pathogenic Photobacterium strains isolated from Exopalaemon carinicauda causing mortality of shrimp. Aquaculture 464: 129-135.

Liu H., Wang, L., Liu, M., Wang, B., Jiang, K., Ma, S., Li, Q., 2011. The intestinal microbial diversity in Chinese shrimp (Fenneropenaeus chinensis) as determined by PCR-DGGE and clone library analyses. Aquaculture 317, 32-36.

Ma X, Sun B, Zhu F (2018) Molecular cloning of Kuruma shrimp Marsupenaeus japonicus endonucleasereverse transcriptase and its positive role in white spot syndrome virus and Vibrio alginolyticus infection. Fish Shellfish Immunol. 73: 297-308.

Md-Zoqratt MZH, Eng WWH, Thai BT, Austin CM, Gan HM (2018) Microbiome analysis of Pacific white shrimp gut and rearing water from Malaysia and Vietnam: implications for aquaculture research and 
management. PeerJ 6: e5826.

Metian M, Troell M, Christensen V, Steenbeek J, Pouil S (2020) Mapping diversity of species in global aquaculture. Rev. Aquacult. 12: 1090-1100.

Nayak S (2010) Probiotics and immunity: A fish perspective. Fish Shellfish Immunol. 29 (1): 2-14.

Nelson TM, Rogers TL, Brown MV (2013) The gut bacterial community of mammals from marine and terrestrial habitats. PLoS One 8 (12): e83655.

Oxley AP, Shipton W, Owens L, McKay D (2002) Bacterial flora from the gut of the wild and cultured banana prawn, Penaeus merguiensis. J. Appl. Microbiol. 93 (2): 214-223.

Pérez-Pascual D, Lunazzi A, Magdelenat G, Rouy Z, Roulet A, Lopez-Roques C, Larocque R, Barbeyron T, Gobet A, Michel G, Bernardet JF, Duchaud E (2017) The complete genome sequence of the fish pathogen Tenacibaculum maritimum provides insights into virulence mechanisms. Front. Microbiol. 8: 1542.

Qi C, Wang L, Jiang K, Wang M, Zhao W, Wang B (2017) Transcriptomic and morphological analyses of Litopenaeus vannamei intestinal barrier in response to Vibrio paraheamolyticus infection reveals immune response signatures and structural disruption. Fish Shellfish Immunol. 70: 437-450.

Qiao F, Liu Y, Sun Y, Wang X, Chen K, Li T, Li E, Zhang M (2017) Influence of different dietary carbohydrate sources on the growth and intestinal microbiota of Litopenaeus vannamei at low salinity. Aquacult. Nutr. 23: 444-452.

Ramírez C and Romero J (2017) Fine flounder (Paralichthys adspersus) microbiome showed important differences between wild and reared specimens. Front. Microbiol. 8: 271.

Ramírez C and Romero J (2017a) The microbiome of Seriola lalandi of wild and aquaculture origin reveals differences in composition and potential function. Front. Microbiol. 8: 1844.

Remany M, Cyriac D, Nagaraj S, Rao B, Panda A, Kumar J, Samraj Y (2010) Specific pathogen-free assurance of imported Pacific white shrimp Litopenaeus vannamei (Boone, 1931 in the Aquatic Quarantine Facility, Chennai. Curr. Sci. 99(12): 1656-1658.

Ringø E, Zhou Z, Vecino JLG, Wadsworth S, Roomero J, Krogdahl Å, Olsen RE, Dimitroglou A, Foey A, Davies S, Owen M, Lauzon HL, Martinsen LL, De Schryver P, Bossier P, Sperstad S, Merrifield DL (2016) Effect of dietary components on the gut microbiota of aquatic animals. A never-ending story? Aquacult. Nutr. 22: 219- 282.

Roeselers G, Mittge EK, Stephens WZ, Parichy DM, Cavanaugh CM, Guillemin K, Rawls JF, (2011) Evidence for a core gut microbiota in the zebrafish. ISME J 5(10): 1595-1608. 
Rungrassamee W, Klanchui A, Chaiyapechara S, Maibunkaew S, Tangphatsornruang S (2013) Bacterial population in intestines of the Black Tiger Shrimp (Penaeus monodon) under different growth stages. PLoS One 8(4): e60802.

Rungrassamee W, Klanchui A, Maibunkaew S, Chaiyapechara S, Jiravanichpaisal P, Karoonuthaisiri N (2014) Characterization of intestinal bacteria in wild and domesticated adult black tiger shrimp (Penaeus monodon). PLoS One 9(3): e91853.

Rungrassamee W, Klanchui A, Maibunkaew S, Karoonuthaisiri N (2016) Bacterial dynamics in intestines of the black tiger shrimp and the Pacific white shrimp during Vibrio harveyi exposure. J. Invert. Pathol. 133: $12-19$.

Sajeela KA, Gopalakrishnan A, Basheer VS, Mandal A, Bineesh KK, Grinson G, Gopakumar SD (2019) New insights from nuclear and mitochondrial markers on the genetic diversity and structure of the Indian white shrimp Fenneropenaeus indicus among the marginal seas in the Indian Ocean. Mol. Phylogenet. Evol. 136: $53-64$.

Schloss PD, Westcott SL, Ryabin T, Hall JR., Hartmann M, Hollister EB (2009) Introducing Mothur: opensource. platform-independent, community-supported software for describing and comparing microbial communities. Appl. Environ. Microbiol. 75: 7537-7541.

Småge SB, Frisch K, Brevikm OJ, Watanabe K, Nylund A (2016) First isolation, identification and characterization of Tenacibaculum maritimum in Norway, isolated from diseased farmed sea lice cleaner fish Cyclopterus lumpus L. Aquaculture 464: 178-184.

Suo Y, Li E, Li T, Jia Y, Qin JG, Gu Z, Chen L (2017) Response of gut health and microbiota to sulfide exposure in Pacific white shrimp Litopenaeus vannamei. Fish Shellfish Immunol. 63: 87-96.

Tarnecki AM, Burgos FA, Ray CL, Arias CR (2017) Fish intestinal microbiome: diversity and symbiosis unraveled by metagenomics. J. Appl. Microbiol. 123: 2-17.

Thompson J, Gregory S, Plummer S, Shields RJ, Rowley AF (2010) An in vitro and in vivo assessment of the potential of Vibrio spp. as probiotics for the Pacific White shrimp, Litopenaeus vannamei. J. Appl. Microbiol. 109: 1177-1187.

Urbanczyk H, Ast JC, Dunlap PV (2011) Phylogeny, genomics, and symbiosis of Photobacterium. FEMS Microbiol. Rev. 35: 324-342.

Vandenberghe J, Verdonck L, Robles-Arozarena R, Rivera G, Bolland A, Balladares M, Gomez-Gil B, Calderon J, Sorgeloos P, Swings J (1999) Vibrios associated with Litopenaeus vannamei larvae, postlarvae, broodstock, and hatchery probionts. Appl. Environ. Microbiol. 65: 2592-2597.

Vaseeharan B, Sundararaj S, Murugan T, Chen J (2007) Photobacterium damselae ssp. damselae associated with diseased black tiger shrimp Penaeus monodon (Fabricius) in India. Let. Appl. 
Microbiol. 45: 82-86.

Velázquez-Lizárraga AE, Juárez-Morales JL, Racotta IS, Villarreal-Colmenares H, Valdes-Lopez O, LunaGonzález A, Rodríguez-Jaramillo C, Estrada N, Ascencio F (2019) Transcriptomic analysis of Pacific white shrimp (Litopenaeus vannamei, Boone 1931) in response to acute hepatopancreatic necrosis disease caused by Vibrio parahaemolyticus. PLoS ONE 14: e0220993.

Wang CZ, Lin GR, Yan T, Zheng ZP, Chen B, Sun FL (2014) The cellular community in the intestine of the shrimp Penaeus penicillatus and its culture environments. Fish Sci. 80: 1001-1007.

Wang J, Uang Y, Xu K, Zhang X, Sun H, Fan L, Yan M (2019) White spot syndrome virus (WSSV) infection impacts intestinal microbiota composition and function in Litopenaeus vannamei. Fish Shellfish Immunol. 84: 130-137.

Wang Q, Garrity GM, Tiedje JM, Cole JR (2007) Naïve Bayesian Classifier for Rapid Assignment of rRNA Sequences into the New Bacterial Taxonomy. Appl. Environ. Microbiol. 73 (16): 5261-5267.

Wei H, Wang H, Tang L, Wang C (2019) High-throughput sequencing reveals the core gut microbiota of the mud crab (Scylla paramamosain) in different coastal regions of southern China. BMC Genom. 20: 829.

Wu S, Wang G, Angert ER, Wang W, Li W, Zou H (2012) Composition, diversity, and origin of the bacterial community in grass carp intestine. PLoS One 7: e30440.

Yan Q, Li J, Yu Y, Wang J, He Z, Van NJD, Zhou J (2016) Environmental filtering decreases with fish development for the assembly of gut microbiota. Environ. Microbiol. 18: 4739-4754.

Zeng S, Huang Z, Hou D, Liu J, Weng S, He J (2017) Composition, diversity and function of intestinal microbiota in pacific white shrimp (Litopenaeus vannamei) at different culture stages. PeerJ 5: e3986.

Zhang, M., Sun, Y., Liu, Y., Qiao, F., Chen, L., Liu, W., Du, Z., Li, E., 2016. Response of gut microbiota to salinity change in two euryhaline aquatic animals with reverse salinity preference. Aquaculture $454,72-$ 80 .

Zhou, J., Fang, W., Yang, X., Zhou, S., Hu, L., Li, X., Qi, X., Su, H., Xie, L., 2012. A nonluminescent and highly virulent Vibrio harveyi strain is associated with "bacterial white tail disease" of Litopenaeus vannamei shrimp. PloS one 7 (2), e29961.

\section{Figures}




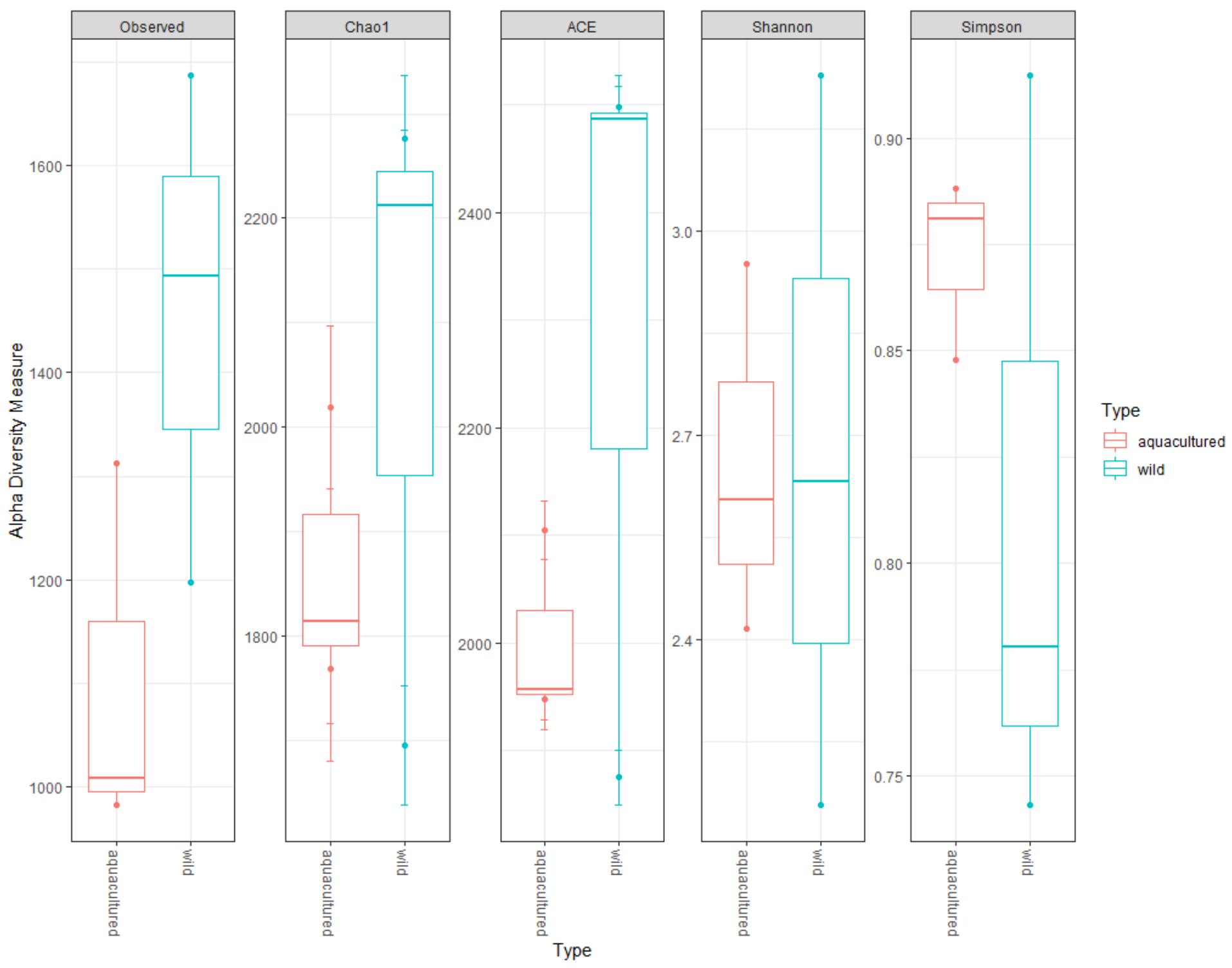

\section{Figure 1}

Comparison of alpha diversity indexes between wild and aquacultured Indian white shrimp (Penaeus indicus). Diversity in the gut bacterial community was measured using Chao-1, Ace, Shannon, Simpson, Fisher indices, which reflects richness and evenness of the samples.
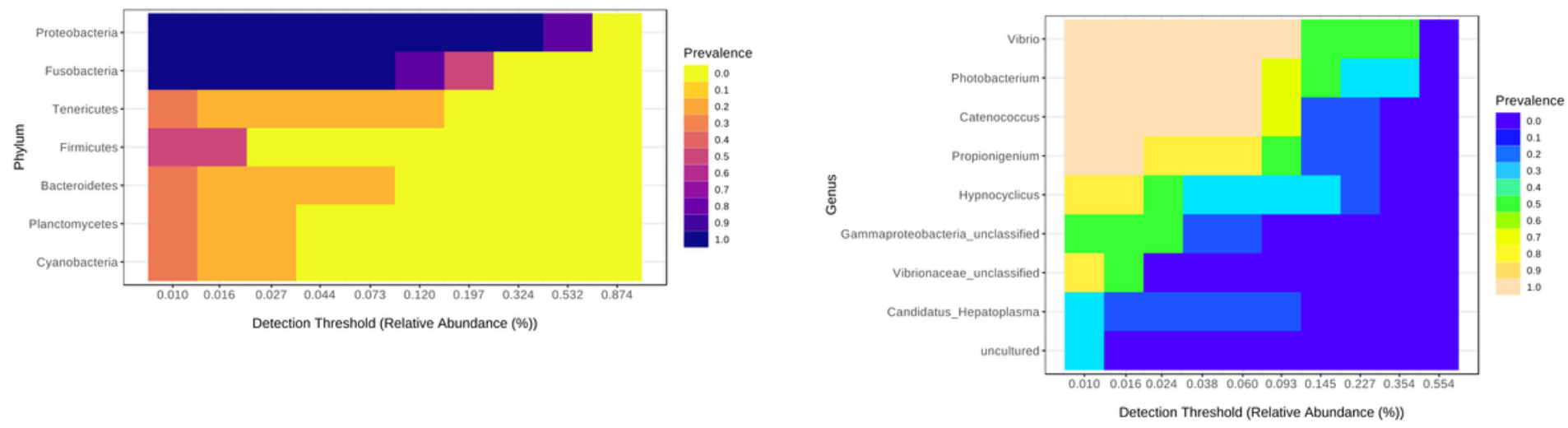
Figure 2

The core gut microbiota of $P$. indicus at phylum (A) and genus (B) level identified by MicrobiomeAnalyst using the parameters sample prevalence $(20 \%)$ and relative abundance $(0.2 \%)$.

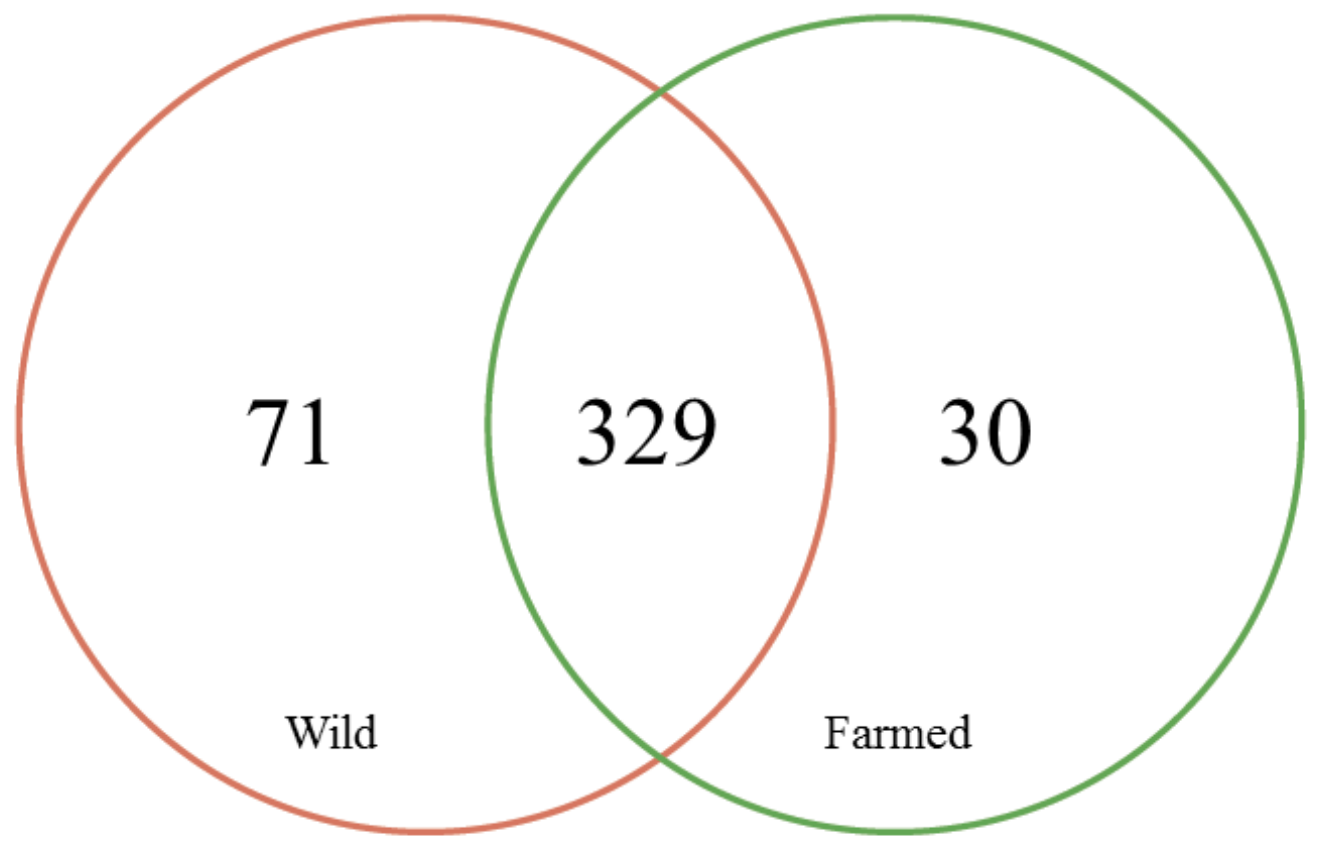

Figure 3

Venn diagram showing the number of OTUs that were unique or shared between wild and aquacultured shrimp. 



Figure 4

Relative abundance of top 10 OTUs at the phylum (A) and genus (B) levels in the gut microbiota from wild and aquaculture $P$. indicus. The relative abundance was calculated based on taxonomy assignment using the Silva database. 